Alkaloide. Die Erklärung dieser Thatsache liegt, wie Mylius, Schiff und Udransky ${ }^{2}$ ) bewiesen haben, darin, dass der hauptsächlichste Factor dieser chromatischen Reactionen das Furfurol ist, welches bei der Einwirkung der Säuren auf die Zuckerarten gebildet wird, und $\mathrm{zwar}$, wie es scheint, am besten und in grösster Menge bei der Einwirkung der Säure auf Lävulose. Man kann daher in der Baudouin'schen Reaction den Zucker oder die Lärulose durch das Furfurol ersetzen, eben wie schon Udransky für die Pettenkofer'sche und Wender für die Alkaloid-Reactionen in Vorschlag gebracht haben. Somit werden einige secundäre Reactionen, die nur störend auf die hauptsächlichste Reaction wirken können, vermieden, und die Baudouin'sche Reaction erhält überdies eine überwiegende Empfindlichkeit.

Da, wie bekannt, das Furfurol für sich mit Salzsäure eine ins Violett spielende Färbung gibt, so ist es nöthig, dasselbe in etwas verdünntem Zustand anzuwenden, in welchem Falle keine Färbung eintritt. Die Lösung, die wir als beste gefunden haben, ist die, welche in 100 cc Alkohol beiläufig $2 \mathrm{~g}$ vom Furfurol (Kahlbaum) gelöst enthält.

Um nun eine Ölmischung, in der man die Gegenwart des Sesamöls im Verdacht hat, zu prüfen, verfährt man wie folgt:

In ein Probirglas bringt man 0,1 cc der hergestellten Furfurollösung, setzt 10 cc des zu prüfenden Öls und dann 10 cc Salzsäure von 1,19 specifisches Gewicht hinzu, schüttelt das Ganze für 1/2 Minute und überlässt dann die Mischung sich selbst. Im Falle das Sesamöl in kleinerer Menge als 1 Proc. sich vorfindet, ist die am Boden des Probirglases sich abscheidende wässerige Lösung entschieden carmoisinroth gefärbt. Fehlt dagegen das Sesamöl ganz, so nimmt die wässerige Lösung keine Farbe an, oder höchstens eine schmutziggelbe Farbe, wie wenn man z. B. mit einem ganz ranzig reinen Olivenöl zu thun hat, die mit jener rothen Färbung, welche das Sesamöl hervorruft, nicht zu verwechseln ist.

Die Probe kann man auch in folgender Art ausführen: In ein Probirglas, welches 0,1 cc der Furfurollösung enthält, setzt man 10 ce des zu prüfenden Öls und dann 1 cc Salzsäure hinzu, man schüttelt gut durch und dann fügt man noch $10 \mathrm{ce}$ Chloroform $\mathrm{zu}$ in der Art, dass alles Öl gelöst wird und beobachtet nun die Farbe der auf dem

2) E. Nickel, ebenda, s. S. 39 und 40.
Chloroform sich abscheidenden wässerigen Flüssigkeit. Auch im Falle, dass das Öl weniger als 1 Proc. vom Sesamöl enthält, nimmt die Flüssigkeit eine schöne carmoisinrothe Färbung an, dagegen färbt sich bei Nichtrorhandensein des Sesamöls die Flüssigkeit nicht, und bei ranzigem oder eine Ausnahme machendem Olivenöle ist nur eine grünliche Färbung zu sehen.

Diese zwei Methoden wurden mit einer sehr grossen Zahl von echten Oliven- sowie von Samen und thierischen Ölen geprüft, und immer konnten wir die Genauigkeit dieses Verfahrens feststellen. Die zur Untersuchung gebrachten Öle waren sehr verschieden. Die Olivenöle kamen aus der Toscana, Liguria, Sicilien, Puglien, der römischen Campagna und aus Tunisi. Die Samenöle aus erster und zweiter Pressung waren folgende: Erdnussöle aus Mozambique Coromandel, Senegal Bombay .., Rapsöle, Colza-, Mandel- und Baumwollsamenöle, Lein-, Nuss-, Mohnöle aus verschiedener Bezugsquelle und endlich als Thieröle dienten Ochsenklauenöle, Walthrane und Fischthrane.

Rom, Laboratorio Centrale della Gabelle.

Mischgas und Generatorgas.

Von

Ferd. Fisoher.

Kürzlich hatte ich Gelegenheit, eine neue Anlage für Mischgas zu untersuchen, welche schlechtes Gas lieferte, weil übermässig Wasserdampf zugeführt wurde.

Für die Vergasung von Kohlenstoff kommen bekanntlich ${ }^{1}$ ) folgende Reactionen in Frage:
1. $\mathrm{C}+\mathrm{O}_{2}=\mathrm{CO}_{2}$
2. $\mathrm{C}+\mathrm{O}=\mathrm{CO}$
$\mathrm{C}+\mathrm{CO}_{2}=2 \mathrm{CO}$
4. $\mathrm{C}+\mathrm{H}_{2} \mathrm{O}=\mathrm{CO}+\mathrm{H}_{2}-39400 \quad 136400$
(Wasserdampf v. $20^{\circ}-28600 \quad 125600$ )
5. $\mathrm{C}+2 \mathrm{H}_{2} \mathrm{O}=\mathrm{CO}_{2}+2 \mathrm{H}_{2}-39800 \quad 136800$
(Wasserdampf v. $20^{\circ}-28200 \quad 125200$ )

Wärmetönung $\begin{gathered}\text { Brennwerth } \\ \text { der erhalt. }\end{gathered}$

Bei der Vergasung von Kohlenstoff durch atmosphärische Luft nach Gleichung 2 erfordern somit $12 \mathrm{k}$ Kohlenstoff $11,15 \mathrm{cbm}$ Sauerstoff (in Mischung mit $41,95 \mathrm{cbm}$ Stickstoff), welche $22,3 \mathrm{cbm}$ Kohlenoxyd geben,

1) Vergl. d. Z. 1887, II, 147; Fis cher's Jahresb. 1887, 157; Fischer: Taschenbuch für Feuerungstechniker, 2. Aufl., S. 8. 
so dass aus $53,1 \mathrm{cbm}$ Luft $64,2 \mathrm{cbm}$ Gas folgender Zusammensetzung:

$$
\begin{aligned}
& \text { Kohlenoxyd 34,7 Proc. } \\
& \text { Stickstoff } \quad 65,3 \quad \text { - }
\end{aligned}
$$

entstehen; je unvollkommener die Generatorgasbildung ist, um so mehr nähert sich die Umsetzung der Gleichung 1.

Bei der vollkommenen Geueratorgasbildung (2) werden 29000 W. E. frei, welche dem entweichenden Gase eine Temperatur von etwa $1500^{\circ}$ ertheilen (von Wärmeverlusten des Generators abgesehen). Wo daber das Gas nicht mit dieser hohen Temperatur verwendet werden kann, ist es vortheilhaft, diese Wärme so viel als möglich zur Gasbildung nach Gleichung 3, 4 oder 5 zu verwenden. Diese Reactionen erfordern aber eine Temperatur von annähernd $1000^{\circ}$, welche daher im Generator zu erhalten ist. Hierzu sind aber rd. 19000 W.E. erforderlich, so dass nur 10000 in dieser Richtung verwerthbar bleiben. (Vgl. S. 525 d. Z.)

Bei Einfübrung von Wasserdampf $f^{2}$ ) kann man demnach für je dreimal $\mathrm{C}+\mathrm{O}$ einmal Gleichung 4 oder 5 einsetzen

$$
4 \mathrm{C}+3 \mathrm{O}+\mathrm{H}_{2} \mathrm{O}=4 \mathrm{CO}+\mathrm{H}_{2}
$$

$4 \mathrm{C}+3 \mathrm{O}+2 \mathrm{H}_{2} \mathrm{O}=\mathrm{CO}_{2}+3 \mathrm{CO}+2 \mathrm{H}_{2}$ somit für $48 \mathrm{k}$ Kohlenstoff:

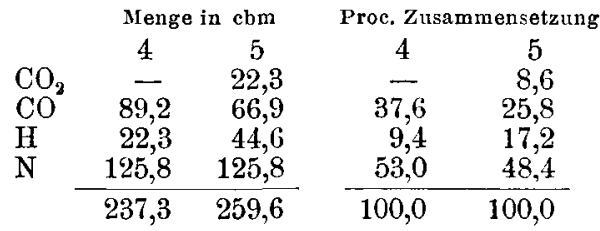

$48 \mathrm{k}$ Kohlenstoff geben demnach 237 bez. $259 \mathrm{cbm}$ Gas von 329400 bez. 320000 W. E. ${ }^{3}$ ), entspr. 85 Proc. Ausnutzung gegen 70 Proc. beim einfachen Generatorgas.

Letztere Zusammensetzung (5) entspricht dem früher von mir aus einer Anlage der Körting'schen Fabrik untersuchten Mischgas (d. Z. 1891, 693). Werden Luft und Dampf vorgewärmt, so wird entsprechend mehr Wasser zersetzt werden können, desgl. wenn die Reaction theilweise nach Gleichung 1 verläuft, was sich durch höheren Gehalt an Kohlensäure und Wasserstoff zeigt, wie bei den Analysen von Trillich (S. 286 d. Z.). Die Zusammensetzung des Gases wird sich im Allgemeinen um so mehr der unter 4 angegebenen nähern, je gleichmässiger die Temperatur auf etwa $1000^{\circ}$ gehalten wird.

2) Bei Dampfstrahlgebläsen ist Dampf bereits vorhanden, sonst wird er durch Abhitze erzeugt.

3) Wasserdampf von $20^{\circ}$ als Verbrennungsprod. S. 66 des Taschenbuches ist in der Tabelle Jetzte Spalte leider ein Satzfehler stehen geblieben: der Brennwerth von $1 \mathrm{cbm}$ Kohlenoxyd ist selbstverständlich auch auf Wasserdampf bez. 3050 , nicht 5050 .
Ganz wird sie der unvermeidlichen Wärmeverluste des Apparates wegen ohne Vorwärmung von Luft und Dampf nicht erreicht werden können, während Gleichung 5 auch bei etwas niederer Temperatur verläuft, wenn die Apparate genügend gross sind.

Nach Gleichung 2 und 4 sind demnach auf je $160 \mathrm{cbm}$ Luft $18 \mathrm{k}$ Wasserdampf, nach 2 und 5 sind $36 \mathrm{k}$ Wasserdampf erforderlich; wird mehr zugeführt, so wird Wärme weggeführt, die Temperatur im Generator herabgesetzt und in Folge dessen schlechtes Gas erzeugt.

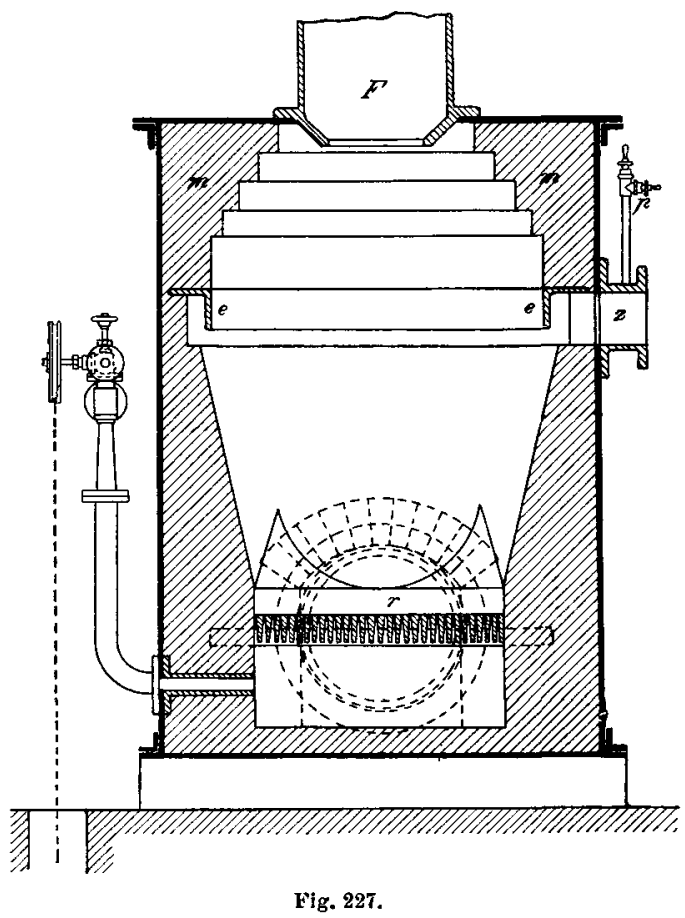

Der erwähnte, erst vor wenig Wochen von H. in I. gelieferte Apparat für Mischgas hat, wie Fig. $227(1 / 20)$ zeigt, eine von den sonst gebräuchlichen ${ }^{4}$ ) abweichende Form. Der Kohlenraum erweitert sich über dem Rost $r$ bis zum Gasabzug $z$, Eisenring $e$ hält den ringförmigen Gassammelraum von deu durch Fülltrichter $F$ eingeführten Kohlen frei und trägt das Mauerwerk $m$. Diese Vorrichtung verhindert die Erhitzung der Decke (vgl. S. 286 d. Z.), vermindert also den Wärmeverlust und soll wohl eine Zersetzung der Destillationsproducte bezwecken.

Dem Hahn $p$ entnommene Gasproben enthielten bei Untersuchung mit dem kleinen Gasapparat $^{5}$ ) 15 Proc. Kohlensäure. Beim Einschmelzen von Proben schied das Gas

4) Fischer: Taschenbuch für Fenerungstechniker, 2. Aufl., S. 15.

5) Fischer: Taschenbuch für Feuerungstechniker, 2. Aufl., S. 39. Die damit ausgeführten Analysen sind mit einem $*$ bezeichnet. 
in den Glaskugeln sehr viel Wasser $a b$; es wurde also offenbar übermässig Wasserdampf zugeführt. Die Untersuchung dieser Proben (vgl. S. 512) ergab:

$\begin{array}{rrrrrr}\text { Zeit } & \mathrm{CO}_{2} & \mathrm{CO} & \mathrm{CH}_{4} & \mathrm{H} & \mathrm{N} \\ 11 \mathrm{U} .30 & * 15,0 & - & - & - & - \\ 11-40 & 15,1 & 8,8 & 1,0 & 20,3 & 54,8 \\ 11-45 & * 14,9 & - & - & - & - \\ 11-55 & 14,8 & 8,8 & 0,6 & 20,1 & 55,7 \\ 12-\frac{-}{2} & * 15,2 & - & - & - & - \\ 2-10 & * 15,1 & - & - & - & - \\ 2-15 & 14,9 & 8,6 & 1,9 & 22,5 & 52,1 \\ 2-25 & * 15,2 & - & - & - & -\end{array}$

In der Mittagspause wurde abgeschlackt und neu beschickt. Der verwendete engl. Anthracit (angebl. Gwaun Cal Gurwen) enthielt trocken:

$\begin{array}{lc}\text { Kohlenstoff } & 92,31 \text { Proc. } \\ \text { Wasserstoff } & 3,07 \\ \text { Sauerstoff }(+\mathrm{N}+\mathrm{S}) & 1,52 \\ \text { Asche } & 3,10\end{array}$

Die sog. Immediatanalyse ergab:

$\begin{array}{lr}\text { Wasser } & 2,60 \\ \text { Fluchtige Stoffe } & 7,13 \\ \text { Asche } & 3,01 \\ \text { Aschefr. Koks } & 87,26\end{array}$

Abgerundet ist die mittlere Zusammensetzung des Gases:

$\begin{array}{lc}\text { Kohlensäure } & 15 \text { Proc. } \\ \text { Kohlenoxyd } & 9 \\ \text { Methan } & 1 \\ \text { Wasserstoff } & 21 \\ \text { Stickstoff } & 54\end{array}$

Abgesehen von dem bei der Entgasung gebildeten Methan und Wasserstoff hat somit die Vergasung nach den Gleichungen 1, 2 und 5 etwa zu gleichen Theilen stattgefunden:

$$
3 \mathrm{C}+3 \mathrm{O}+2 \mathrm{H}_{2} \mathrm{O}=2 \mathrm{CO}_{2}+\mathrm{CO}+2 \mathrm{H}_{2} \text {. }
$$

Durch den übermässig zugeführten Wasserdampf ist die Temperatur im Gaserzeuger so niedrig gehalten, dass sie für die Reaction nach Gl. 4 nicht ausreichte. $1 \mathrm{cbm}$ Gas hatte einen Brennwerth von nur 1092 W. E., war demnach recht mangelhaft; thatsächlich konnte die 25 pferd. Maschine nicht damit ordentlich betrieben werden, umsomehr die mitgeführten Theernebel Schieber u. dgl. verschmierten. Die Temperatur war zu niedrig, um die Theerdämpfe zu zersetzen, so dass die Abführung des Gases in der Mitte den Zweck verfehlte; übrigens ist die Haltbarkeit des Ringes $e$ fragwürdig. Für vorliegenden $Z_{\text {weck }}$ erscheint der Gaserzeuger zu klein.

Ein Dampfstrahlgebläse erfordert zum Einführen einer bestimmten Luftmenge umsomehr Dampf, je grösser der zu überwindende Widerstand ist. Dieser hängt aber ab von der Beschaffenheit der Rohrleitungen, der Kohlenschicht, den Reinigern $u$. s. w.
Das Verhältniss zwischen Luft und Dampf wechselt auch, wenn beim Heben der Gasometerglocke der Dampf gedrosselt wird und hängt noch besonders vom Dampfdruck und von der Construction des Gebläses ab, alles Umstände, welche die gleichmässige Lieferung eines guten Gases in Frage stellen. Der jetzt erforderliche Dampfkessel zum Betriebe des Gebläses vertheuert Anlage und Betrieb, so dass ein Absaugen des Gases u. U. vortheilhafter sein würde; beim Maschinenbetrieb z. B. könnte die Saugrorrichtung unmittelbar von der Maschine betrieben werden, so dass nur für Inbetriebsetzung eine Hülfsvorrichtung (Handgebläse, kleiner Gasometer o. dgl.) erforderlich wäre. Die Wasserzufuhr würde durch den Gasverbrauch geregelt, die Verdampfung geschähe im Wärmeausgleicher, in den die erforderjiche Luft frei eintreten kann.

Die Verwendung von Kohlensäure würde sich ebenso stellen, als von flüssigem Wasser, wie Gl. 3 bis 5 zeigen; man kann daher auf $4(\mathrm{C}+\mathrm{O})$ nur $1 \mathrm{CO}_{2}$ verwenden:

$$
5 \mathrm{C}+2 \mathrm{O}_{2}+\mathrm{CO}_{2}=6 \mathrm{CO}
$$

Das erzeugte Gas hat demnach folgende $\mathrm{Zu}-$

\begin{tabular}{|c|c|c|}
\hline \multirow{2}{*}{$\begin{array}{l}\text { Kohlenoxyd } \\
\text { Stickstoff }\end{array}$} & $133.8 \mathrm{cbm}$ & 44,3 Proc. \\
\hline & 167,8 & 55 \\
\hline
\end{tabular}
sammensetzung:

$60 \mathrm{k}$ Kohlenstoff geben somit $301,6 \mathrm{cbm}$ Gas von 408000 W.E., entspr. 84 Proc. Ausnutzung; die Beschaffung von Kohlensäure für Gaserzeugung kann daher nicht lohnend sein.

Siemens verwendet kohlensäurehaltige Verbrennungsgase (d. Z. 1890, 19 u. 144), führt daher mit $22,3 \mathrm{cbm}$ Kohlensäure etwa $90 \mathrm{cbm}$ Stickstoff ein. Das Gas würde daher folgende Zusammensetzung haben:

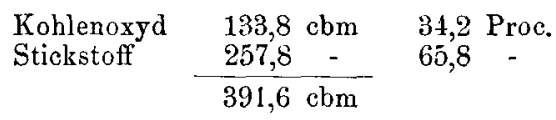

Thatsächlich wird man ohne starke Vorwärmung der Gase diese Zusammensetzung nie erreichen können, da die Erhitzung der $90 \mathrm{cbm}$ Stickstoff auf die Temperatur des Gaserzeugers $\left(1000^{\circ}\right) 27000$ W. E. erfordert, so dass weit über die Hälfte der verfügbaren Wärme hierzu verbraucht würde. $54 \mathrm{k}$ Kohlenstoff würden daher günstigstensfalls liefern können:

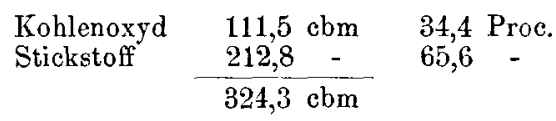

von 340000 W. E. Brennwerth, entspr. nur 78 Proc. Ausnutzung. Bez. Ausnutzung der Kohle im Gaserzeuger stellt sich daher die 
Vergasung durch Einführung von Wasserdampf entschieden günstiger als die Verwendung von kohlensäurehaltigen Verbrennungsgasen.

Für die Ausnutzung des Brennwerthes dieses letzteren Gases ( $3 \mathrm{a}$ ) in Vergleich zu den nach Gl. 2, 4 u. 5 erzeugten sind folgende Verbältnisse beachtenswerth:
$60 \mathrm{k}$ Kohlenstoff geben dann mit $159 \mathrm{cbm}$ Luft und 36 bez. $72 \mathrm{k}$ Wasserdampf

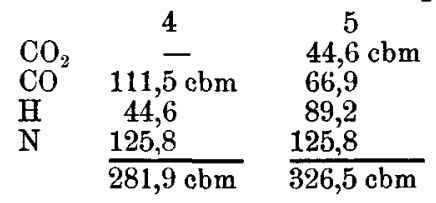

\begin{tabular}{|c|c|c|c|c|}
\hline & 2 & $3 a$ & 4 & 5 \\
\hline 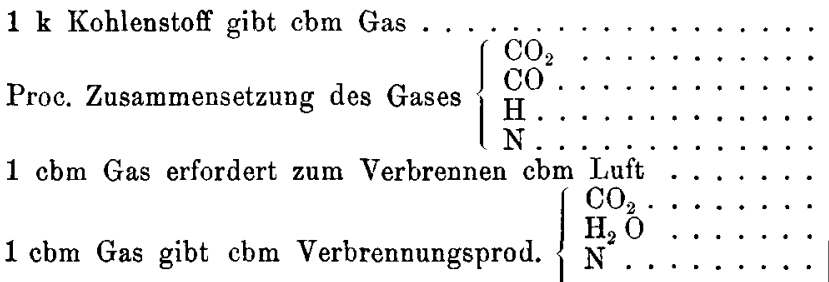 & $\begin{array}{l}5,35 \\
-\overline{34,7} \\
- \\
65,3 \\
0,83 \\
0,35 \\
\overline{1,31}\end{array}$ & $\begin{array}{l}\frac{6,01}{34,4} \\
\overline{-1} \\
65,6 \\
0,82 \\
0,34 \\
\overline{1,31}\end{array}$ & $\begin{array}{l}4,94 \\
- \\
37,6 \\
9,4 \\
53,0 \\
1,12 \\
0,38 \\
0,09 \\
1,41\end{array}$ & $\begin{array}{r}5,41 \\
8,6 \\
25,8 \\
17,2 \\
48,4 \\
1,02 \\
0,34 \\
0,17 \\
1,29\end{array}$ \\
\hline Zusammen & 1,66 & 1,65 & 1,88 & 1,80 \\
\hline $\begin{array}{l}\text { Brennwerth von } 1 \text { ebm Gas (Wasserdampf von } 20^{\circ} \text { bez.) } . \\
\text { Davon verwendb., wenn d. Verbrennungspr. }{ }^{6} \text { ) mit } 1000^{0} \text { entw. } \\
\text { Desgl. bez. auf } 1 \text { k Kohlenst. . . . . . . . . . . . }\end{array}$ & $\begin{array}{r}1058 \\
477 \\
2552\end{array}$ & $\begin{array}{r}1049 \\
471 \\
2831\end{array}$ & $\begin{array}{r}1389 \\
731 \\
3611\end{array}$ & $\begin{array}{r}1231 \\
601 \\
3251\end{array}$ \\
\hline
\end{tabular}

Die Einführung von Verbrennungsgasen (3a) in Gaserzeuger ist demnach viel ungünstiger als die von Wasserdampf. Da ferner nur wasserstoffhaltige Gase leicht brennen, so kann für die Erzeugung von Mischgas, besonders für Maschinenbetrieb, nur die Miteinführung von Wasserdampf in Frage kommen; die Zusammenstellungen zeigen, dass möglichst die Zersetzung nach Gleichung 4 anzustreben ist.

Ein Theil der Eigenwärme des gebildeten Gases wird zur Vorwärmung des Anthracits o. dgl. (sp. W. $=0,4)$ und zur Entgasung bez. Verdampfung der Feuchtigkeit verwendet, ein grösserer Theil geht durch den Apparat verloren, besonders wenn derselbe mit Wasser bedeckt ist. Würde die Decke gewölbt, z. B. nach Fig. 227, so würde letzterer Verlust wesentlich geringer. Würde man dann das Gas durch eine Wärmeausgleichvorrichtung ${ }^{7}$ ) führen, das Dampfluftgemisch aber entgegengesetzt, so könnte man letzteres auf $500^{\circ}$ bis $550^{\circ}$ vorgewärmt unten in den Vergaser eintreten lassen. Man würde dann etwa doppelt soviel Wärme verfügbar haben bez. Wasserdampf einführen können als vorhin: $5 \mathrm{C}+3 \mathrm{O}+2 \mathrm{H}_{2} \mathrm{O}=5 \mathrm{CO}+2 \mathrm{H}_{2}$ $5 \mathrm{C}+3 \mathrm{O}+4 \mathrm{H}_{2} \mathrm{O}=2 \mathrm{CO}_{2}+3 \mathrm{CO}+4 \mathrm{H}_{2}$

6) Wegen der ansteigenden spec. Wärme der Kohlensäure ist die durchschnittl. spec. Wärme zu 0,35 angenommen, über $1000^{\circ} \mathrm{zu} 0,4$; vgl. Fis cher: Taschenbuch für Feuerungstechniker, 2. Aufl., S. 53.

7) Bei einer Vorrichtung, wie sie z. B. Fig. 87 S. $171 \mathrm{~d}$. Z. andeutet, würde das Gas die Röhren umspülen, durch welche das Luft-Dampfgemisch entgegengeführt würde; das Gas würde hier schon Theer, Flugstaub u. dergl. absetzen. von 455200 bez. 434400 W. E. (Wasserd. v. $20^{\circ}$ bez.), somit 94 bez. 90 Proc. Ausnutzung, ferner:

$\begin{array}{llll}1 \mathrm{k} \text { Kohlenst. gibt cbm Gas } & 4 & 5 \\ \text { Proc. Zus. der Gase } & 4,70 & 5,44 \\ & & - & 13,7 \\ \text { Brennw. von 1 cbm W. E. } & & 1612 & 1330\end{array}$

Die Vorwärmung des Luftdampfgemisches hat somit einen sehr günstigen Einfluss auch auf die Zusammensetzung des Gases, so dass die dafür erforderlichen Anlagekosten sich bald bezahlt machen würden. Das Gas wäre auch ein gutes Heizgas für chemische Fabriken.

Für Gasfeuerungen, welche hohe Hitzegrade erfordern, ist eine Vorwärmung auf etwa $1000^{\circ}$ wohl erreichbar. Es würde dann die gesammte Wärme der Reaction 2 in chemische Arbeit umgesetzt werden können, so dass für einmal $\mathrm{C}+\mathrm{O}$ auch einmal Gleichung 4 (5 kommt für diese Temperatur nicht in Frage) und für $4 \mathrm{C}+\mathrm{O}$ fast dreimal Gleichung 3 angesetzt werden kann. Hierzu reine Kohlensäure zu beschaffen ist von vornherein aussichtslos, so dass nur Verbrennungsgase (20 Proc. $\mathrm{CO}_{2}$ ) in Frage kommen könnten:

$$
\text { Menge }\left\{\begin{array}{lcc}
\mathrm{CO}_{2} & 3 \mathrm{a} & \mathbf{4} \\
\mathrm{CO} & 223,0 & \overline{44,6} \\
\mathrm{H} & \overline{-} & 22,3 \\
\mathrm{~N} & \frac{435,4}{658,4} & \frac{42,0}{108,9}
\end{array}\right.
$$

$1 \mathrm{k}$ Kohlenst. $=\mathrm{cbm} \mathrm{Gas}$ 


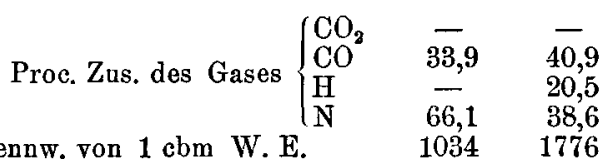

Wenn nun Gas und Verbrennungsluft mit derselben Temperatur in den Ofen treten, mit welcher die Verbrennungsgase denselben verlassen, so wird offenbar die gesammte Wärme der vergasten Kohle im Ofen ausgenutzt, soweit sie nicht durch das Mauerwerk verloren geht. Praktisch ist dieses aber nicht möglich, vielmehr müssen die Gase den Ofen stets viel heisser verlassen, als sie bei Durchführung der besprochenen Vergasungsverfahren eintreten können. Wenn Gas und Luft mit $1000^{\circ}$ eintreten, die Verbrennungsgase mit $1400^{\circ}$ austreten, so ergibt sich:

\begin{tabular}{|c|c|c|c|}
\hline 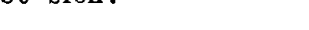 & & & \\
\hline $\begin{array}{l}1 \text { cbm Gas gibt } \\
\text { ebm Verbrennungsgas }\end{array}$ & $\left\{\begin{array}{l}\mathrm{CO}_{2} \\
\mathrm{H}_{2} \mathrm{O} \\
\mathrm{N}^{2}\end{array}\right.$ & $\frac{0,34}{1,30}$ & $\begin{array}{l}0,41 \\
0,20 \\
1,54\end{array}$ \\
\hline $\begin{array}{l}\text { anw. v. } 1 \\
\text { ivon ver }\end{array}$ & & $\begin{array}{r}1,64 \\
1034 \\
772\end{array}$ & $\begin{array}{r}2,15 \\
1776 \\
1432\end{array}$ \\
\hline
\end{tabular}

Um die gleiche Wärmemenge in den Ofen zu bringen, hat man also doppelt so viel von dem mit Kohlensäuregasen erzeugten Gas (3a) nöthig, als von dem mit Wasserdampf erzeugten. Dementsprechend werden auch für ersteres etwa doppelt so grosse Apparate erforderlich sein, entsprechend auch der unvermeidliche Wärmeverlust an die Umgebung, so dass das durch Einführen von Verbrennungsgasen erzeugte Gas noch ungünstiger ist, als obige Zusammenstellung zeigt.

Bei der neuen Siemens'schen Feuerung wird ein Theil der Verbrennungsgase durch ein Dampfstrahlgebläse wieder unter den Rost geführt (d. Z. 1890, 19). Dass die a. a. O. darüber gemachten Angaben falsch sind, wurde bereits (d. Z. 1890, 144) nachgewiesen und die vorstehenden Berechnungen zeigen, dass die Einführung von bis zu $1000^{\circ}$ heissen Verbrennungsgasen recht unvortheilhaft ist. Es bleibt also nur noch die Frage, $o b$ es vortheilhaft sein kann, noch heissere Gase einzuführen.

Die Reaction $\mathrm{C}+\mathrm{CO}_{2}=2 \mathrm{CO}$ erfordert 39000 W. E.; die $22,3 \mathrm{cbm}$ Kohlensäure sind mit rd. $90 \mathrm{cbm}$ Stickstoff gemischt, zusammen also $112 \mathrm{cbm}$ Verbrennungsgase. Nimmt man die sp. W. derselben über $1000^{\circ}$ selbst zu 0,4 an, so würden die Gase $900^{\circ}$ heisser dem Generator zugeführt werden müssen, als zur Reaction erforderlich ist, also mit etwa $1900^{\circ}$, was doch praktisch unausführbar ist. Si emens bläst gleichzeitig Wasserdampf ein, dessen Erbitzung und Zersetzung ebenfalls erhebliche Wärmemengen erfordert. Ohne Luftzufuhr ist dieses Verfahren somit ganz unmöglich. Die Reaction

$$
2 \mathrm{C}+\mathrm{CO}_{2}+\mathrm{H}_{2} \mathrm{O}=3 \mathrm{CO}+\mathrm{H}_{3}
$$

erfordert 68000 W. E. Würden nun die Verbrenuungsgase mit $1400^{\circ}$ zum Generator geführt, so liefern diese 17900 W. E., während die Erhitzung des Wasserdampfes auf $1000^{\circ} 8800$ W. E. erfordert. Es würden also noch 59000 W. E. durch die Reaction $\mathrm{C}+\mathrm{O}=\mathrm{CO}=29000$ zu decken sein. Nehmen wir nun den günstigsten Fall, die Luft könnte mit etwa $1200^{\circ}$ zugefübrt werden, so hätte man

$$
4 \mathrm{C}+\mathrm{CO}_{2}+\mathrm{H}_{2} \mathrm{O}+\mathrm{O}_{2}=5 \mathrm{CO}+\mathrm{H}_{2} \text {, }
$$

somit für $48 \mathrm{k}$ Kohlenstoff $112 \mathrm{cbm}$ Verbrennungsgase, $18 \mathrm{k} \mathrm{Wasserdampf}$ und $106 \mathrm{cbm}$ atmosphärische Luft das gebildete Gas:

\begin{tabular}{lll} 
CO & 111,5 cbm & 36,3 Proc. \\
H & 22,4 & 7,3 \\
N & 173,1 & 56,4 \\
\cline { 2 - 3 } & 307,0 &
\end{tabular}

Brennwerth von $1 \mathrm{cbm}$ Gas $=1295 \mathrm{~W} . \mathbf{E}$.

Selbst das unter so günstigen Umständen erhaltene Gas stellt sich sonach erheblich ungünstiger als das nur mit Wasserdampf erzielte S. 509; thatsächlich wird das Ergebniss der unvermeidlichen Wärmeverluste wegen noch schlechter sein.

Die Einführung von Verbrennungsgasen in die Gaserzenger ist daher in jeder $\mathrm{Be}$ ziehung eine Verschlechterung, das Kreisen der grossen Stickstoffmengen immer mit grossen Wärmeverlusten verbunden. Wenn - wie in Reclamen behauptet wird - durch dieses Verfahren wirklich 50 Proc. Brennstoffersparung gegen früher erzielt werden sollte, so würde dieses nur beweisen, dass die früheren Anlagen noch um soviel schlechter waren. -

Für Generatorgasfeuerungen genügt die von den Verbrennungsgasen fortgeführte Wärme vollständig, sowohl die Vergasungsluft wie auch die Verbrennungsluft auf etwa $1000^{\circ}$ vorzuwärmen. Liegt dann der Gaserzeuger unmittelbar unter bez. bei der Verwendungsstelle, wie z. B. in den Leuchtgasanstalten, so ist theoretisch die Verwendung von Luft allein am vortheilhaftesten. Die Hitze im Gaserzeuger würde dann aber sehr hoch, so dass das Mauerwerk stark angegriffen würde. Man verwendet daher meist kalte oder nur wenig vorgewärmte Vergasungsluft. Besser würde es in diesem Falle sein, Wasserdampf einzuführen, wodurch die Hitze im Gaserzeuger ermässigt wird und im Verbrennungsraum auch gleichmässiger zur Wirkung kommt, nur ist dann um so mehr darauf zu sehen, dass von den abziehenden Ver- 
brennungsgasen möglichst viel Wärme zur Vorwärmung von Dampf und Luft verwerthet wird. Dieses geschieht am besten in Wärmeausgleichvorrichtungen (Regeneratoren) mit Gegenströmung ${ }^{8}$ ), viel weniger gut in den Siemens'schen Wärmespeichern ${ }^{9}$ ).

Bei grössern Anlagen wäre wohl zu erwägen, ob es nicht lohnt, die überschüssige Hitze zur Dampferzeugung o. dgl. zu verwenden. Lässt man das Gas mit der Temperatur des Generators in den Ofen treten, so braucht nur die Luft vorgewärmt $z u$ werden. Für $1 \mathrm{cbm}$ Generatorgas (bestes) sind nach S. 509 nur 0,83 cbm Luft erforderlich, während 1,66 cbm Verbrennungsgase entweichen. Diese entführen (bei $1200^{\circ}$ ) 700 W. E., während zur Vorwärmung der Luft auf $1000^{\circ}$ nur 250 W. E. erforderlich sind.

Die Regenerirung der Hochofengase, welche von J.v.Ehrenwerth (Fischer's J. 1884, 1300) empfohlen wurde, stellt sich insofern etwas günstiger wie die Verwendung von Verbrennungsgasen, als jene bereits viel Koblenoxyd enthalten. Ehrenwerth übersieht aber bei seinen Berechnungen, dass in dem von ihm beschriebenen Gaserzeuger annähernd $1000^{\circ}$ erforderlich sind.

Angenommen, das Gichtgas habe die dort angegebene Zusammensetzung (abgerundet):

$\begin{array}{lr}\mathrm{CO}_{2} & \mathbf{1 6} \\ \mathrm{CO} & 23 \\ \mathrm{H} & 3 \\ \mathrm{~N} & 58\end{array}$

so würden zur Ausfübrung der Reaction

$$
\mathrm{C}+\mathrm{CO}_{2}=2 \mathrm{CO}
$$

auf $12 \mathrm{k}$ Kohlenstoff $140 \mathrm{cbm}$ Gichtgase erforderlich sein, oder bei der von Ehrenwerth rorgeschlagenen 2/3-Reduction $r d$. $200 \mathrm{cbm}$. Um diese auf etwa $1000^{\circ} \mathrm{zu}$ erhitzen, sind rd. 43000 bez. 60000 W. E. erforderlich; letzterer Vorschlag ist demnach ungünstiger als die vollständige Regenerirung. Es sei daher nur diese angenommen, als Wärmeverlust nur 5000 W. E. (Ehrenwerth selbst nimmt 30 Proc. an), so würde auf obige Gleichung dreimal Gleichung 2 erforderlich sein:

$$
4 \mathrm{C}+3 \mathrm{O}+\mathrm{CO}_{2}=5 \mathrm{CO}
$$

$48 \mathrm{k}$ Kohlenstoff geben dann:

\begin{tabular}{lrrr} 
& CO & \multicolumn{1}{c}{ N } & H \\
$3 \mathrm{C}+3 \mathrm{O}=$ & 66,9 & 125,9 & - \\
Gichtgase & 76,8 & 81,2 & 4,2 \\
\cline { 2 - 4 } & 143,7 & 207,1 & 4,2
\end{tabular}

somit $355 \mathrm{cbm}$ Gas folgender Zusammensetzung:

8) Vgl. Ferd. Fischer: Handbuch der chemischen Technologie, 14. Aufl. S. 96, 102 u. 753.

${ }^{9}$ ) Vgl. daselbst S. 94 u. 750.

$\begin{array}{lr}\mathrm{CO} & 40,5 \\ \mathrm{H} & \mathbf{1 , 2} \\ \mathrm{N} & 58,3\end{array}$

Brennwerth von $1 \mathrm{cbm}$ Gas $=1266 \mathrm{~W}$. E. gegen 778 W. E. für 1 cbm Gichtgas. Die $355 \mathrm{cbm}$ Gas haben einen Brennwerth von rd. 450000 W. E., die $48 \mathrm{k}$ Kohlenstoff von 388000 W. E.; die 140 cbm Gichtgase hatten 109000 W. E., von denen also nur 62000 ausgenutzt wurden. Praktisch wird sich die Sache noch wesentlich ungünstiger stellen, so dass dieses Verfahren nur da in Frage kommen kann, wo die Hochofengase sonst ungenutzt verloren gehen, während gutes Generatorgas gewünscht ist. Aber selbst in diesen seltenen Fällen dürfte es vortheilhafter sein, die Gichtgase zu verbrennen, damit das verwendete Luftdampfgemisch auf $500^{\circ}$ oder mehr zu erhitzen; um ein Gas zu erzielen, wie es unter 4 S. 509 besprochen wurde und zur Erzielung hoher Temperaturen oder als Kraftgas viel vortheilhafter ist. Wenn überhaupt die Regenerirung der Gichtgase schon irgendwo durchgeführt ist, so werden die Erfolge nicht gross sein, sonst würden bereits entsprechende Veröffentlichungen vorliegen. Für bez. Mittheilungen wäre ich dankbar. -

Die Angabe von Trillich (Bayer. Ind. Gew. 1893, 101), eine Untersuchung von Mischgas dauere mindestens 2 Stunden, veranlasst mich $\mathrm{zu}$ folgenden Bemerkungen:

Bei dem früher (d. Z. 1891, 694) beschriebenen Apparat wird der Schlauchansatz $a$ jetzt seitlich an das Absorptionsgefäss $A$ (Fig. 228) angeschmolzen, damit der Zünder $g$ mitten durch die untere Verschraubung hindurchgeführt werden kann. Es ist besonders auf guten Schliff der drei Glashähne zu sehen, sowie darauf, dass der obere Theil von $A$ die Form von d. Nebenfig. habe, damit sich an der Abzweigung $c$ des zum Messrohr $M$ führenden Hahnrohres kein Tropfen halten kann, der dann leicht die Hähne und $M$ verunreinigen würde. Dass die Führung des Zünders $g$ sowie die Verbindung $v$ dicht schliessen müssen, ist selbstverständlich. Verwendet man für den Zünder $g$ zum Glühendmachen der Platinspirale 2 gute Kohle-Zink-Salmiakelemente (oder 3 Trockenelemente), so glüht die Spirale hinlänglich lange, ohne Gefahr des Abschmelzens, so dass die Einschaltung eines Widerstandes nicht erforderlich ist.

Bei Ausführung der Analyse zieht man Zünder $g$ nach unten (so dass die Drahtschlinge in den unteren Theil von $A$ kommt), füllt die Rohre $A, M, D$ durch Heben der beiden Flaschen $F$ und $L$ mit Quecksilber, schliesst die drei Glashähne, so dass das Schlauchende des Dreiweghahnes $d$ mit Wasser (oder Quecksilber) gefüllt 
bleibt, steckt das eine ausgezogene Ende der die Gasprobe enthaltenden zugeschmolzenen Glaskugel hinein, bricht mit einer Flachzange die Spitze innerhalb des Schlauches $a b$, taucht das andere Ende in ein kleines Becherglas mit Wasser (oder Quecksilber), bricht auch hier die Spitze ab, dreht Hahn $d$ so, dass die Verbindung mit $\boldsymbol{A}$ hergestellt ist, und saugt die Gasprobe durch Senken der Quecksilberflashe $F$ nacb $A$ herüber ${ }^{10}$ ). Nun dreht man Hahn $d$ und $h$ um $90^{\circ}$ und treibt durch

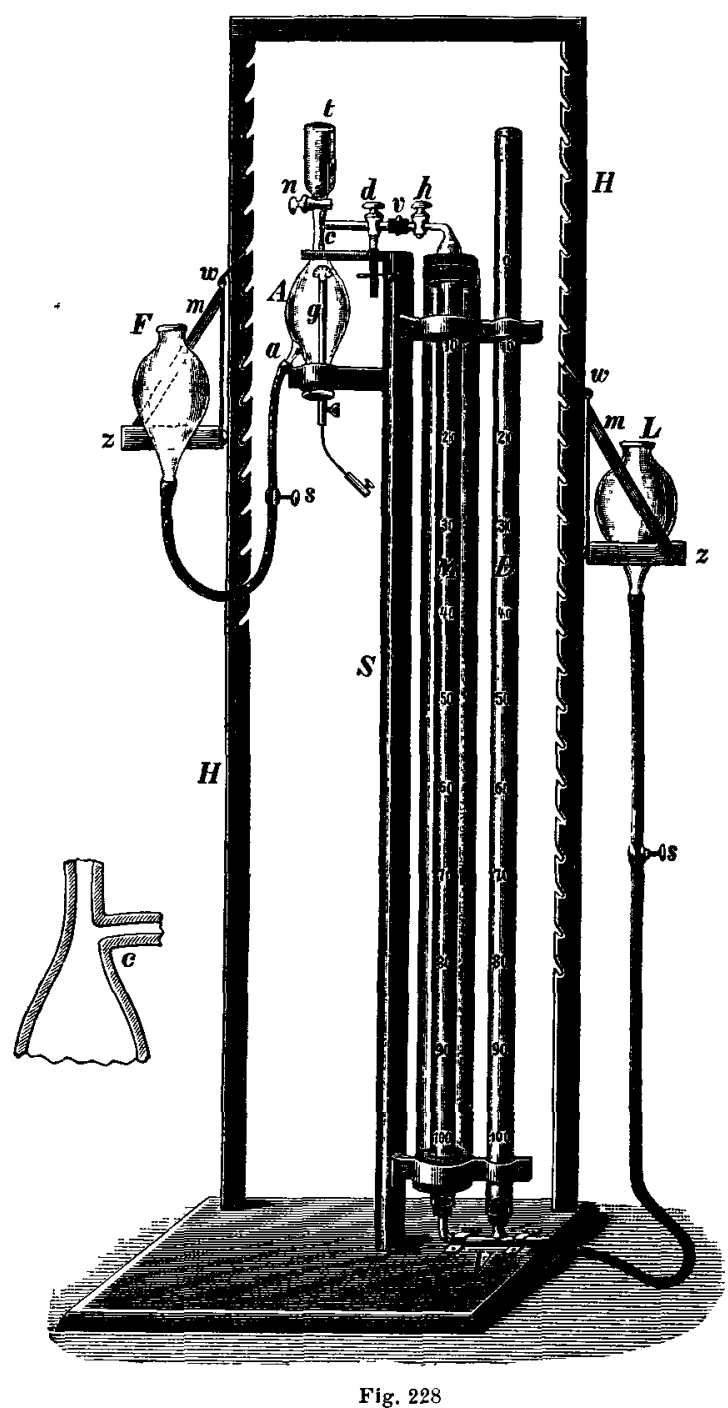

Heben der Quecksilberflasche $F$ und Senken der anderen Flasche $L 45$ bis 50 cc des Gases in das Messrohr $M$. Ist im Behälter $A$ ein Gasrest und etwa übergesogenes Wasser enthalten, so drückt man nach Schliessung des Hahnes $h$ diese durch Hahn $d$ und angehängten Schlauch in eine Sammelflasche. Nun wird die Gasprobe gemessen, durch Trichter $t$ etwa 0,5 cc Kalilauge in Be-

10) Die Arbeit wird Ungeubten erleichtert, wenn sie die beiden Schraubenquetschbahne $s$ so einstellen, dass das Quecksilber zum Aufsteigen bez. Fallen in den Röhren $M D 1$ bis 2 Minuten gebraucht. hãlter $\boldsymbol{A}$ gelassen, $n$ geschlossen, dann die Gasprobe aus $M$ nach $A$ übergeführt, nach der Bindung der Kohlensãure (etwa 2 Minuten) wieder nach $M$; man lässt dabei aber die Kalilauge nur bis zu einer Marke unmittelbar vor $d$ steigen, welche bei der Calibrirung des Rohres berücksichtigt werden kann. Ist so Kohlensăure bestimmt (und bei unsicheren Proben auf Sauerstoff mit Pyrogallol geprüft), so muss Behälter $A$ bis Hahn $d$ sorgfältig gereinigt werden, da sonst nach der Verbrennung ein Theil der gebildeten Kohlensäure sofort gelöst wird. Dieses gesehieht durch Eingiessen von etwa 10 bis $20 \mathrm{cc}$ Wasser in den Trichter $t$, Senken der Flasche $F$, dann Heben derselben, so dass die Flussigkeit durch Hahn $d$ in eine (durch ein kurzes Glasrohr und längeren Schlauch damit verbundene) Flasche abfliesst. Dieses wird 4 bis 5 mal wiederholt.

Zur Verbrennung des Wasserstoffs, Kohlenwasserstoffes und Kohlenoxyds lässt man durch Hahn $n$ etwa 60 cc Luft in den Behälter $A$ treten (den man passend mit einer Marke versehen hat), schliesst $n$, senkt $L$, hebt $F$, offnet $d$ und $h$, so dass die Luft nach $M$ ubertritt, bis die Gesammtmenge des Gasgemisches rd. $100 \mathrm{cc}$ beträgt. $h$ wird geschlossen, dann wird abgelesen, der etwaige Luftrest durch Heben von $F$ aus $A$ entfernt. Hahn $n$ wird dann gescblossen, $d$ und $h$ so gestellt, dass beim Heben der Flasche $L$ und Senken von $F$ das Gasgemisch in den Behälter $A$ tritt. Sobald die Platinspirale des Zünders $g$ aus dem Quecksilber herausragt, schliesst man den Strom, so dass die Spirale eben rothglühend wird, und treibt rasch den Rest des Gases ein. Der Vorsicht wegen drickt man das Gas noch einmal nach $M$, bis das Quecksilber eben die Spirale berührt, sofort nach $A$ zurück, löst die Stromverbindung, zieht $g$ zurück, drückt das Gas nach $M$ und liest ab. Nun bringt man durch Trichter $t$ etwa 0,5 cc Kalilauge in $A$, führt das Gas ein, nach etwa 2 Minuten zurück nach $M$ und liest ab. Dann lässt man etwa 0,3 ce Pyrogallussäurelösung $(1: 3)$ zn der Kalilauge nach $A$, darauf das Gas aus $M$; zur Beschleunigung der Absorption lässt man etwa 2 Minuten lang von $M$ nach $A$ Quecksilber übertreten, dessen herunterfallende Tropfen Gas und Absorptionsflüssigkeit fortwährend mischen. Das Gas wird nach $M$ zurückgefuthrt und gemessen. Die Analyse ist damit beendet.

Wenn man das mit weitem Wassermantel umgebene Rohr $M$ während der Arbeit nicht beruhrt oder anhaucht und die Temperatur des Laboratoriums ziemlich gleich bleibt, so ändert sich die Temperatur des Gases während der Analyse nicht nennenswerth, wie ein oben in den Wassermantel gestecktes Thermometer zeigt. Stellt man ferner vor jeder Ablesung das Quecksilber in den Rohren $M$ und $D$ gleich hoch (Ablesung mit Loupe), so ist für technische Zwecke jede Correction bei der Berechnung überflüssig.

Ein Versuch ergab z. B.:

Angewandte Gasmenge

Nach Absorption von $\mathrm{CO}_{2} \quad 42,6$

Nach Zulassen von Luft $\quad 99,2$

Nach der Verbrennung $\quad 80,8$

Nach Absorption von $\mathrm{CO}_{2} \quad \mathbf{7 5 , 9}$

Desgl. von Sauerstoff $\quad 72,2$ 
Die zugelassenen $56,6 \mathrm{cc}$ Luft enthalten $44,7 \mathrm{cc}$ Stickstoff, die Gasprobe daher $72,2-44,7=27,5 \mathrm{cc}$, somit $42,6-27,5=15,1$ cc brennbare Gase (V). Da die durch Verbrennung bewirkte Contraction $\mathrm{n}=18,4$, die gebildete Kohlensäure $\mathrm{k}=4,9$, so ergibt sich in bekannter Weise (Taschenbuch S. 49):

\begin{tabular}{lcc} 
Kohlensäure & $\mathbf{7 , 6}$ cc & 15,1 Proc. \\
Kohlenoxyd & 4,4 & 8,8 \\
Methan & 0,5 & 1,0 \\
Wasserstoff & 10,2 & 20.3 \\
Stickstoff & 27.5 & 54,8 \\
\cline { 2 - 3 } & $\overline{50,2}$ & 100,0
\end{tabular}

Bei einiger Übung kann man so eine bis auf $1 / 5$ Proc., also für alle technische $Z$ wecke genügend genaue Analyse in etwa $1 / 2$ Stunde ausführen ${ }^{11}$ ). Für die gewöhnliche Betriebsaufsicht kann man die Vorrichtung noch dahin vereinfachen, dass $D$ fehlt und $L M$ mit Wasser gefüllt wird. - Für wissenschaftlich genaue Analysen ist Ablesen mit Fernrohr, Berechnung auf $0^{\circ}$ und $1000 \mathrm{~mm}$ u. s. w. beizubehalten.

\section{Über Butteruntersuchung.}

Von

\section{Dr. Eduard Spaeth.}

(Mittheilung aus dem Laboratorium der kgl. Untersuchungsanstalt Erlangen.)

Die Bestimmung der einzelnen Bestandtheile der Butter, als welche bei der Beurtheilung ibrer Marktfähigkeit der Gehalt an Wasser, an Fett, an Nichtfett und auch an Kochsalz in Betracht kommen, ist nach den bestehenden Methoden als eine etwas umständliche, öfters auch ungenaue Resultate gebende zu bezeichnen.

Nach den fast allgemein angenommenen, so auch in den Vereinbarungen bayerischer Vertreter der angewandten Chemie angegebenen Normen der Beurtheilung darf eine Butter von guter Beschaffenheit nicht über 15 Proc. Nichtfett enthalten; eine solche mit mehr als 20 Proc. ist nicht mehr als marktfähig zu erachten. Zur Feststellung des Gehaltes an Nichtfett sind die Bestimmungen des Wassers, dann des Fettgehaltes ausreichend.

Die Bestimmungen des Wassergehaltes werden gewöhnlich in der Weise ausgeführt, dass in einer mit ausgeglühtem Sand halbgefüllten Platinschale 5 bis $10 \mathrm{~g}$ Butter abgewogen und dann 1/2 Stunde lang auf dem kochenden Wasserbade und später im Trockenschranke bei etwa $100^{\circ}$ getrocknet werden,

11) Meinen Apparat fertigte Universitätsmechaniker W. Apel in Göttingen an. oder es werden etwa $10 \mathrm{~g}$ Butter in einem Glasgefäss 6 Stunden lang unter Umschwenken bei $100^{\circ}$ getrocknet.

Zur Bestimmung des Fettgehaltes werden etwa $5 \mathrm{~g}$ Butter in einer Porzellanschale geschmolzen und mit $20 \mathrm{~g}$ Gyps gemischt, dann 6 Stunden lang bei $100^{\circ}$ getrocknet und das nach dem Erkalten trockene Pulver mit absolutem Äther im Extractionsapparate bis zur Erschöpfung behandelt; anstatt des Gypses allein sind auch Mischungen von Sand und Gyps empfohlen; das Trocknen geschieht auch in Hoffmeister'schen Schälchen.

Nun haben zahlreiche, doppelt ausgeführte Bestimmungen des Wasser- und Fettgehaltes in einer Butter äusserst selten zu übereinstimmenden Resultaten geführt, was auch leicht erklärlich wird, da das Wasser oder die Buttermilch, die bei der Bereitung der Butter nicht genügend entfernt oder die, was recht häufig zu beobachten ist, durch verschiedene Bearbeitung der Butter einverleibt werden, nie gleichmässig in der Butter vertheilt sein können. Es sind daher, indem zur Bestimmung des Wassergehaltes einerseits und des Fettgehaltes andererseits nach den angegebenen Untersuchungsmethoden verschiedene Mengen Butter in Anwendung gezogen wurden, aus den erhaltenen Zablen des Wassers und des Fettes ganz unrichtige Schlüsse auf den Gehalt an Nichtfett (d. h. Caseïn, Albumin, Salze) gefolgert worden, wie nachstehend verzeichnete Resultate zeigen.

\begin{tabular}{r|c|c|c|c|c|c}
\hline \hline & \multicolumn{2}{|c|}{ Wassergehalt } & \multicolumn{2}{|c|}{ Fettgehalt } & \multicolumn{2}{|c}{ Nichtfett } \\
& $\mathbf{A}$ & $\mathbf{B}$ & $\mathbf{A}$ & $\mathbf{B}$ & $\mathbf{A}$ & $\mathbf{B}$ \\
\hline I & 17,25 & $\mathbf{1 5 , 9 5}$ & 76,99 & 82,33 & 5,76 & 1,72 \\
II & 17,17 & 16,10 & 79,10 & 78,90 & $\mathbf{3 , 7 3}$ & $\mathbf{5 , 0 0}$ \\
III & $\mathbf{1 2 , 0 9}$ & 11,69 & 86,50 & 86,40 & 1,41 & 1,91 \\
IV & 12,94 & 10,59 & 86,30 & 88,30 & 0,76 & 1,11 \\
V & 16,90 & 16,80 & 82,00 & 83,00 & 1,10 & 0,20 \\
VI & 23,56 & 21,40 & 74,20 & 75,30 & 2,24 & 3,30
\end{tabular}

Um diese an und für sich nicht angenehmen Methoden, besonders die Methode der Fettbestimmung, zu umgehen und um es möglich zu machen, in einer abgewogenen Buttermenge Wasser, Fett, Kochsalz bestimmen und so zu sicheren Resultaten kommen zu können, wurde von mir bei Butteruntersuchungen nachstehende Methode, die in einem vollkommen einfachen Apparate zur Ausführung kommt, in Anwendung gezogen.

Der Apparat (Fig. 229) besteht aus einem Glasschiffchen in einer nur etwas grösseren Form, wie dieselben aus Blech von $\mathrm{H}$. Vogel (Dingl. 237, 59) zur Untersuchung der Milch, zur Bestimmung des Trockenrückstandes und des Fettes empfohlen wurden, und aus einem Glasgefäss, das sich von einem gewöhnlichen 